Check for updates

Cite this: Phys. Chem. Chem. Phys., 2020, 22, 13431

Received 12th March 2020 Accepted 31st May 2020

DOI: $10.1039 / \mathrm{d} 0 \mathrm{cp01396j}$

rsc.li/pccp

\section{Quantum-chemistry-aided identification, synthesis and experimental validation of model systems for conformationally controlled reaction studies: separation of the conformers of 2,3-dibromobuta-1,3-diene in the gas phase $\dagger$}

\author{
Ardita Kilaj, (DD ${ }^{a}$ Hong Gao, (D) ${ }^{\text {ab }}$ Diana Tahchieva, ${ }^{a}$ Raghunathan Ramakrishnan, (D) ac \\ Daniel Bachmann, ${ }^{a}$ Dennis Gillingham, ${ }^{a}$ O. Anatole von Lilienfeld, (D) ad \\ Jochen Küpper (D) ${ }^{\text {efgh }}$ and Stefan Willitsch (D) *a
}

\begin{abstract}
The Diels-Alder cycloaddition, in which a diene reacts with a dienophile to form a cyclic compound, counts among the most important tools in organic synthesis. Achieving a precise understanding of its mechanistic details on the quantum level requires new experimental and theoretical methods. Here, we present an experimental approach that separates different diene conformers in a molecular beam as a prerequisite for the investigation of their individual cycloaddition reaction kinetics and dynamics under single-collision conditions in the gas phase. A low- and high-level quantum-chemistry-based screening of more than one hundred dienes identified 2,3-dibromobutadiene (DBB) as an optimal candidate for efficient separation of its gauche and s-trans conformers by electrostatic deflection. A preparation method for DBB was developed which enabled the generation of dense molecular beams of this compound. The theoretical predictions of the molecular properties of DBB were validated by the successful separation of the conformers in the molecular beam. A marked difference in photofragment ion yields of the two conformers upon femtosecond-laser pulse ionization was observed, pointing at a pronounced conformer-specific fragmentation dynamics of ionized DBB. Our work sets the stage for a rigorous examination of mechanistic models of cycloaddition reactions under controlled conditions in the gas phase.
\end{abstract}

\section{Introduction}

Besides polar and radical reactions, pericyclic processes are one of the three fundamental reaction types that form the basis of synthetic organic chemistry. Given their great significance in

\footnotetext{
${ }^{a}$ Department of Chemistry, University of Basel, 4056 Basel, Switzerland. E-mail: stefan.willitsch@unibas.ch

${ }^{b}$ Beijing National Laboratory for Molecular Sciences, Institute of Chemistry, Chinese Academy of Sciences, Beijing 100190, China

${ }^{c}$ Centre for Interdisciplinary Sciences, Tata Institute of Fundamental Research, Hyderabad 500107, India

${ }^{d}$ National Center for Computational Design and Discovery of Novel Materials (MARVEL), University of Basel, 4056 Basel, Switzerland

${ }^{e}$ Center for Free-Electron Laser Science, Deutsches Elektronen-Synchrotron DESY, 22607 Hamburg, Germany

${ }^{f}$ Department of Physics, Universität Hamburg, 22761 Hamburg, Germany

${ }^{g}$ Department of Chemistry, Universität Hamburg, 20146 Hamburg, Germany

${ }^{h}$ Center for Ultrafast Imaging, Universität Hamburg, 22761 Hamburg, Germany

$\dagger$ Electronic supplementary information (ESI) available: Synthesis of 2,3-dibromobutadiene. See DOI: 10.1039/d0cp01396j
}

organic synthesis, rigorously defined mechanistic pathways are an important resource for reaction developers. The Diels-Alder cycloaddition, ${ }^{1}$ in which a diene and a dienophile react to form a cyclic product, is a practical and widely used pericyclic reaction in organic synthesis. While the broad strokes of its mechanism are well understood, the detailed reaction manifold for any given substrate is often extensively discussed. ${ }^{2-7}$ Given its mechanistic subtleties and its importance, the Diels-Alder reaction has served as a test-bed for establishing new types of mechanistic analysis. ${ }^{8}$ In case of the "canonical" concerted pathway, which involves a cyclic transition state and is widely discussed in the literature, ${ }^{2-7}$ only the s-cis conformer of the diene reacts to form the cyclic product - while in a stepwise mechanism also the s-trans conformer can contribute to the reaction. Stepwise pathways become particularly important for ionic variants of the reaction, i.e., polar cycloadditions, for which traditional concepts for rationalizing the mechanism such as the conservation of orbital symmetry break down. ${ }^{4,7,9,10}$ Thus, an experimental investigation of the mechanistic impact 
of individual rotamers is clearly warranted. This, however, requires a way to probe the reactivities of the individual conformers of a specific diene, a difficult task under standard liquid-phase reaction conditions.

In recent years, molecular beams have become an important tool for the investigation of gas-phase chemical reaction dynamics under highly controlled conditions. ${ }^{11,12}$ In particular, the use of inhomogeneous electric fields has enabled the electrostatic deflection and thus spatial separation of different molecular conformers and isomers according to their different electric dipole moments. ${ }^{13-17}$ The combination of such a "controlled" molecular beam with a stationary reaction target of sympathetically cooled molecular ions in an ion trap forms a powerful tool for studies of the kinetics and dynamics of ionmolecule reactions. ${ }^{12,16-18}$ Recently, this approach has enabled the measurement of individual chemical reactivities of the cis and trans conformers of 3-aminophenol with trapped $\mathrm{Ca}^{+}$ ions $^{16,18}$ and of the two nuclear-spin isomers of water ${ }^{19}$ toward trapped diazenylium ions $\left(\mathrm{N}_{2} \mathrm{H}^{+}\right)$in a proton-transfer reaction. ${ }^{17}$ Consequently, molecular beams in conjunction with ion traps offer a direct and precise way to measure conformerspecific rate constants and thus to investigate the reaction mechanism of polar cycloadditions. The key challenge is the identification of suitable model systems amenable to a characterisation under these specific experimental conditions. In the present context, this means that (i) both reactants, the diene and the dienophile, need to be volatile enough to enable their preparation in the gas phase, (ii) the energy difference between the s-cis and s-trans conformers of the diene needs to be small enough so that both can be populated in the cold environment of a supersonic molecular beam, and (iii) the difference of their permanent dipole moments in the molecular frame needs to be large enough to enable their efficient electrostatic separation. ${ }^{11,16}$

The selection of an optimal model system is, therefore, a multi-dimensional optimization problem. Traditionally, the choice would be guided by chemical and physical insight. Nowadays, numerical simulations of reactions can provide meaningful atomistic insights to support experimental efforts. $^{20}$ In the context of designing experiments, virtual screening has proven to be a powerful approach for suggesting compounds matching the physical and chemical properties of interest. Computational screening has already been successfully applied in protein, materials and catalytic design. ${ }^{21-23}$ Here, we apply this approach to identify optimal dienes suitable for controlled gas-phase polar cycloaddition reactions.

This article combines methods of theoretical, organic, and physical chemistry to lay the foundations for a subsequent experimental characterization of conformational effects in polar cycloaddition reactions. Quantum-chemical calculations were performed to screen the reactant space for a diene with physical properties optimized toward its use in conformerselected reaction-dynamics experiments in the gas phase. A synthesis for the theoretically identified optimal diene, 2,3-dibromobutadiene (DBB), was then developed. Finally, the physical properties of the compound were validated in a molecular-beam experiment separating the two conformers by electrostatic deflection.

\section{Theoretical and experimental methods}

\subsection{Theoretical screening}

We applied the concept of computational screening towards the problem of exploring chemical space from first principles ${ }^{24}$ in order to find a polar diene for conformer-selective DielsAlder cycloadditions. The chemical role of polar dienes in Diels-Alder reactions was already explored computationally in various preceding studies, see, e.g., ref. 10 and 25. Efficient electrostatic separation necessitates a certain difference in electric dipole moments $\Delta \mu$ between the s-cis and s-trans conformers of the diene. ${ }^{11,26}$ Moreover, a small energy difference $\Delta E$ between the ground states of the conformers is required to ensure a significant thermal population of both species in the molecular beam. For a successful experiment, a suitable diene has to be identified which satisfies both of these conditions.

High-throughput based virtual design of novel compounds typically starts from an initial scaffold, which can easily be modified at multiple sites through functionalization by substituting atoms or functional groups. ${ }^{27}$ Theoretical screening then yields the best mutated combinations selected according to their proximity to the desirable physical or chemical targetproperty values.

In the present work, we computationally searched the chemical space of butadiene derivatives for which the s-cis and s-trans isomers exhibit maximal and minimal differences in dipole moments and energies, respectively. Substituting $\mathrm{CH}_{2}$ in positions 1 and 4 by $\mathrm{NH}$, or $\mathrm{O}$, and substituting the hydrogen attached to carbon in position 2 and 3 by halogens ( F, Cl, Br, I), a preliminary density functional theory (DFT) based scan of 144 candidates, not accounting for symmetrically redundant species, resulted in the identification of di-halogen substituted butadiene as a promising series of candidates for experiments. Due to the chemical reactivity of iodine-substituted compounds, potentially hampering subsequent synthetic efforts, we have only included the difluoro, dichloro, and dibromo 2,3-substituted butadienes for further in-depth theoretical analysis.

Torsional energy profiles were subsequently calculated for all three species using DFT with the double-hybrid functional DSD-PBEP86-D3BJ ${ }^{28}$ and a large basis set (def2-QZVPP) ${ }^{29}$ which was previously shown to give good performance for the prediction of torsional potential energy surfaces of similar molecules. ${ }^{30}$ For the torsional profiles, the geometry optimizations were restricted by keeping the torsional angle $\Theta=\Theta_{\mathrm{H} 3 \mathrm{C}-}$ CC- $Y$ constant, imposing achirality. The entire range of $0^{\circ}<$ $\Theta<180^{\circ}$ was scanned in steps of $\Delta \Theta=20^{\circ}$. Note that due to the applied constraints for the torsional angles throughout the geometry optimization, the torsional profile is symmetric $\left[E\left(360^{\circ}-\Theta\right)=E(\Theta)\right]$. Calculations were carried out with the Gaussian09 program package. ${ }^{31}$ 


\subsection{Stark-energy and trajectory simulations}

To theoretically assess the behaviour of DBB in an electrostatic deflection experiment, Stark energies and effective dipole moments of individual rotational states of DBB were calculated using the CMIstark software package. ${ }^{32}$ The calculated rotational constants and dipole moments as listed in Table 2 were used as input parameters. The Stark energies served as input parameters for simulating state-specific deflection profiles of the molecular beam by the electrostatic deflector with a homemade software package based on CMIfly. ${ }^{11}$

Trajectory simulations were carried out for gauche-DBB with $10^{5}$ molecules for each rotational state up to a maximum rotational quantum number of $J_{\max }=20$. For the apolar s-trans-DBB, only a single quantum state, $J=0$, needed to be simulated with a total number of $10^{6}$ trajectories. In all cases, initial positions were uniformly sampled across the cross section of the orifice of the gas nozzle generating the molecular beam. The initial velocities of the molecules were sampled from a normal distribution. The velocity distribution was matched to the experimentally determined mean longitudinal velocity of $843 \mathrm{~m} \mathrm{~s}^{-1}$ with a longitudinal velocity spread of $10 \%$. A transverse velocity spread of $4 \mathrm{~m} \mathrm{~s}^{-1}$ was chosen to match the divergence of the beam to the acceptance angle of the skimmers in the assembly. According to the theoretical energy difference between the ground states of gauche- and s-trans-DBB in Table 2, the ratio of their thermal populations at room temperature is $p_{\text {gauche }} / p_{\text {trans }}=0.30$, taking into account the two-fold degeneracy of the gauche structure, see Fig. 2. This ratio was used to scale the simulated deflection profiles of the two species.

In order to calculate thermally averaged deflection profiles $n_{\sigma, T}(y)$, i.e., the beam density $n$ at a specific deflection coordinate $y$, for each conformer $(\sigma \in\{$ gauche,s-trans $\})$ at a specific rotational temperature $T$, we followed a similar procedure as before. ${ }^{17,33}$ For each rotational quantum state $\left|J_{K_{\mathrm{a}} K_{\mathrm{c}}} M\right\rangle$, histograms of the arrival positions $n_{\sigma J K_{\mathrm{a}} K_{\mathrm{c}} M}(y)$ normalized by the initial sample size were extracted from the simulated trajectories. Here, $J$ is the quantum number of the angular momentum neglecting nuclear spin, i.e., for the rotation, $K_{\mathrm{a}}$ and $K_{\mathrm{c}}$ are pseudo-quantum numbers for the projection of the angular momentum onto the $a$ and $c$ molecular axes, and $M$ is the quantum number for the projection of the rotational angular momentum onto the external quantization axis. Thermal averaging was performed using the relation

$$
n_{\sigma, T}(y)=\frac{p_{\sigma}}{N_{\sigma}} \sum_{J=0}^{J_{\max }} \sum_{K_{\mathrm{a}}, K_{\mathrm{c}}} \sum_{M=0}^{J} g_{M} \mathrm{e}^{-E_{J K_{\mathrm{a}} K_{\mathrm{c}}} / k_{\mathrm{B}} T} n_{\sigma J K_{\mathrm{a}} K_{\mathrm{c}} M}(y),
$$

with the partition function

$$
N_{\sigma}=\sum_{J=0}^{J_{\max }} \sum_{K_{\mathrm{a}}, K_{\mathrm{c}}} \sum_{M=0}^{J} g_{M} \mathrm{e}^{-E_{J K_{\mathrm{a}} K_{\mathrm{c}}} / k_{\mathrm{B}} T}
$$

Here, $k_{\mathrm{B}}$ denotes the Boltzmann constant, $p_{\sigma}$ are the populations of the conformers at room temperature and $E_{J K_{\mathrm{a}} K_{\mathrm{c}}}$ are the field-free rotational energies. The degeneracy factor $g_{M}$ takes values $g_{M}=1$ for $M=0$ and $g_{M}=2$ for $M>0$. The total thermal deflection profile was calculated from the sum of the deflection profiles of the gauche- and s-trans-conformers,

$$
n_{\text {tot }, \mathrm{T}}(y)=n_{\text {gauche }, T}(y)+n_{\text {s-trans }, T}(y) .
$$

\subsection{Synthesis of DBB}

Since DBB is an unstable compound which is not commercially available, we needed to devise a synthesis that delivered the material in sufficiently high purity and quantity for molecularbeam experiments. Prior to this work, stewart Jr. et al. ${ }^{34}$ reported a synthesis of DBB from 1,4-dihalo-2-butyne and a cuprous halide which formed activated halide ions present in solution. DBB was obtained through continuous distillation during the reaction. After extensive screening of potential conditions, we found that the elimination reaction of 1,2,3,4tetrabromobutane (TBB) with the sterically hindered base 1,8-diazabicyclo[5.4.0] undec-7-ene (DBU) primarily delivered the elimination product DBB. The most effective conditions involved adding DBU to a solution of TBB in diethyl ether under a constant stream of nitrogen, with $\mathrm{NaI}$ as an additive to accelerate the substitution. Under these conditions, near complete conversion to DBB was observed after 1 hour of reaction time. The purified sample was directly used in the molecular beam apparatus. Further information on the synthesis can be found in the ESI. $\dagger$

\subsection{Experimental setup for conformer separation}

A schematic of the experiment is depicted in Fig. 1. Details of the experimental setup have also been described in our earlier work. ${ }^{16-18,35}$

Briefly, a supersonic jet of DBB seeded in neon was generated using a pulsed gas valve and passed through two skimmers before entering the electrostatic deflector. The resulting molecular beam contained a mixture of the gauche and s-trans conformers of DBB. The inset of Fig. 1 depicts the inhomogeneous electric field in the deflector with a cross marking the nominal molecular beam axis. Here, the two conformers were angularly dispersed and thus spatially separated according to their different dipole moments. ${ }^{11}$ Behind the deflector, the molecular beam was directed at a linearquadrupole ion-trap (LQT) coupled to a time-of-flight mass spectrometer (TOF-MS). The entire molecular beam setup can be tilted vertically with respect to the TOF-MS, which allows probing different regions of the dispersed molecular beam. The tilting angle thus defines a deflection coordinate $y$. When entering the TOF-MS, the DBB molecules were ionized by either pulsed vacuum-ultraviolet (VUV) radiation or femtosecond (fs) laser pulses and accelerated onto a microchannel-plate detector (MCP) using high-voltage electrodes.

2.4.1 Molecular beam. The molecular beam was generated from DBB vapor at room temperature seeded in neon carrier gas at 5 bar. The gas mixture was pulsed through a cantilever piezzo valve (MassSpecpecD ACPV2, $150 \mu \mathrm{m}$ nozzle diameter) at a repetition rate of $10 \mathrm{~Hz}$ and a gas-pulse duration of $250 \mu \mathrm{s}$ at the position of the LQT. The velocity of the molecular beam in 
VUV generation

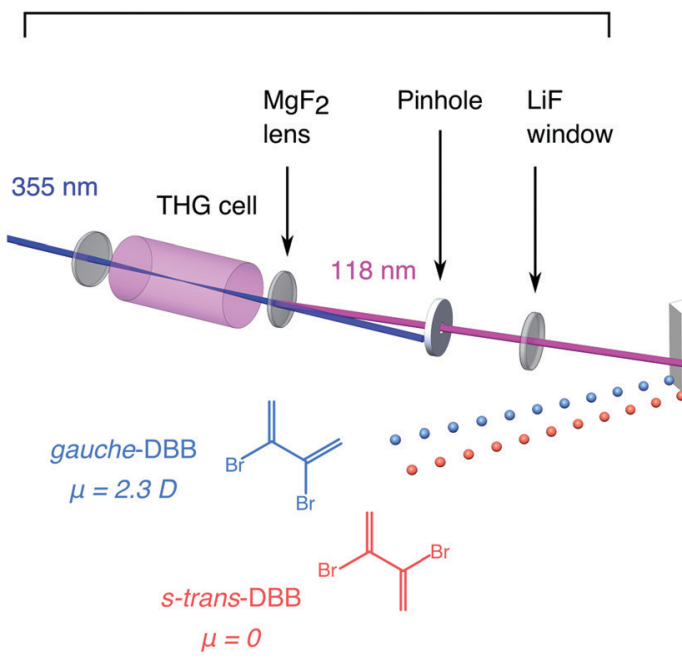

Molecular beam

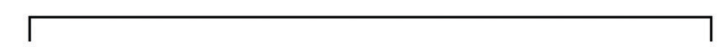

Fig. 1 Overview of the experimental setup.

the direction of propagation beam was measured in the same way as described in Kilaj et al. ${ }^{17}$ and yielded a value of $843(58) \mathrm{m} \mathrm{s}^{-1}$. To determine the density of DBB in the molecular beam, we first calibrated the absolute sensitivity of the TOF-MS by loading Coulomb crystals of defined ion number in our ion trap. ${ }^{36}$ Then, following ref. 37 and 38, we measured the total ion yield of DBB from the molecular beam using ionization with $150 \mathrm{fs}, 775 \mathrm{~nm}$ laser pulses. Observing the logarithmic increase of ion yield with laser intensity ${ }^{38}$ allowed us to estimate a DBB peak density of 7.8(5) $\times 10^{7} \mathrm{~cm}^{-3}$. For deflection experiments, a voltage of $13 \mathrm{kV}$ was applied between the two $15.4 \mathrm{~cm}$ long deflector electrodes, held at a distance of $1.4 \mathrm{~mm}$, to generate the required vertical electric field gradient. ${ }^{11,17,18,33}$ By comparison of the measured deflection profiles with Monte-Carlo simulations, ${ }^{16,17,33}$ a rotational temperature of $1.0 \mathrm{~K}$ could be estimated.

2.4.2 Ion trap and TOF-MS. The LQT is connected to a time-of-flight mass spectrometer (TOF-MS) orthogonal to the molecular-beam propagation axis for quantitative mass analysis. ${ }^{35}$ To enable a better mass selection in the TOF-MS, a voltage of $500 \mathrm{~V}$ was applied to the four end caps of the trap. For extraction of the ionized species, a permanent voltage of $4.0 \mathrm{kV}$ was applied to the repeller electrode. A microchannel plate detector (MCP, Photonis USA) operating at a typical voltage of $2.3 \mathrm{kV}$ was placed at the end of the flight tube.

2.4.3 Femtosecond and VUV ionization. Non-resonant multi-photon ionization of the DBB molecules was performed with pulses from a Ti:Sapphire femtosecond laser (CPA 2110, Clark-MXR, Inc.) at a wavelength of $775 \mathrm{~nm}$ and pulse duration of $150 \mathrm{fs}$ focused to a diameter of $\sim 30 \mu \mathrm{m}$ in the sample. In addition, a vacuum-ultraviolet (VUV) light source was used for soft ionization of the DBB molecules. Similar to other work, ${ }^{39-41}$ pulses of $118 \mathrm{~nm}$ light were generated using thirdharmonic generation (THG) by focusing the third harmonic output beam of a Nd:YAG laser (Quantel Brilliant, $355 \mathrm{~nm}$,
$5 \mathrm{~ns}$ ) into a gas cell containing a phase-matched gas mixture of xenon and argon (ratio 1:10, total pressure 100 mbar). The pump laser was operated at a repetition rate of $10 \mathrm{~Hz}$ and the pulse energy was set to $25 \mathrm{~mJ}$ such that a UV to VUV conversion efficiency of approximately $10^{-5}$ was achieved. The VUV beam was re-focused (spot size $\sim 100 \mu \mathrm{m}$ ) into the trap chamber at the center of the ion trap with a single $\mathrm{MgF}_{2}$ lens (Thorlabs, $f=200 \mathrm{~mm}$ ) at a distance of $120 \mathrm{~mm}$ from the UV-laser focus (spot size $\sim 15 \mu \mathrm{m}$ ). Owing to its stronger index of refraction in the VUV, the $\mathrm{MgF}_{2}$ lens also served as an optical element to separate the pump-laser beam from the VUV beam. A LiF window was used to seal off the ultra-high-vacuum chamber housing the ion trap from the VUV-generation chamber. In order to block the $355 \mathrm{~nm}$ pump-laser beam and prevent it from entering the interaction region or damaging the UV sensitive LiF window, a MACOR-protected pinhole was installed in front of the LiF window. The VUV detector was fabricated from two copper electrodes with a typical bias voltage of about $1 \mathrm{kV}$ and the VUV-induced photocurrent was measured through the resulting voltage across a $50 \Omega$ resistor.

\section{Results and discussion}

\subsection{Torsional profiles of the 2,3-dihalobutadienes}

A graphical representation of the calculated torsional profiles of the 2,3-difluoro-, dichloro-, and dibromobutadienes is shown in Fig. 2a. The global minimum was found to be the s-trans structure (at $\Theta=180^{\circ}$ ) in all cases. Local minima were found to be near gauche (rather than s-cis) structures at torsional angles varying from $\Theta=50^{\circ}$ to $60^{\circ}$ depending on the specific molecule.

Subsequently, differences in potential energy and absolute dipole moment between the local and global minima were calculated including harmonic and anharmonic thermal corrections of zero-point vibrational energy and Gibbs free energy. 
a)

b)
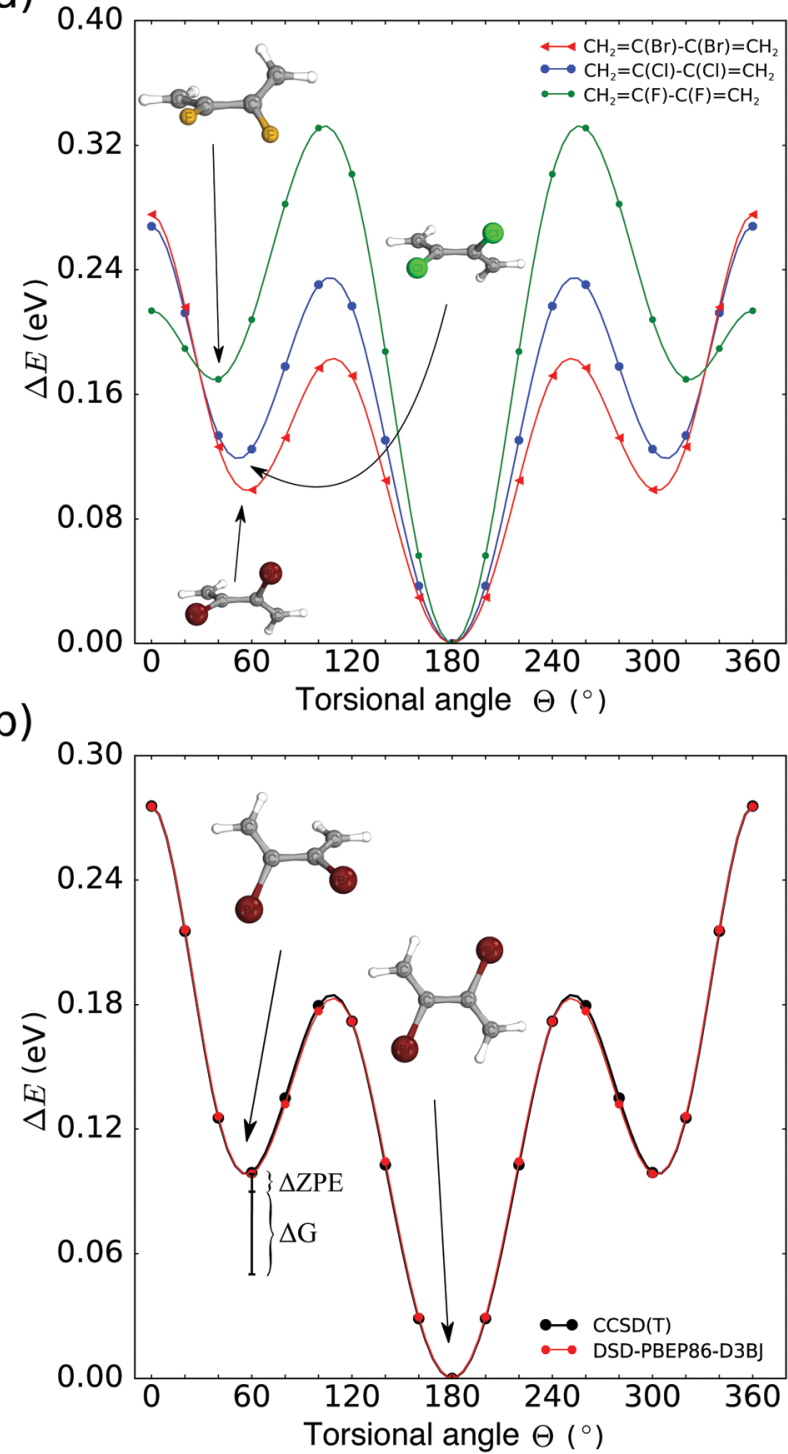

Fig. 2 (a) Cuts through the potential energy surface of 2,3-dibromo1,3-butadiene $\left(\mathrm{CH}_{2}=\mathrm{C}(\mathrm{Br})-\mathrm{C}(\mathrm{Br})=\mathrm{CH}_{2}\right), \quad$ 2,3-dichloro-1,3-butadiene $\left(\mathrm{CH}_{2}=\mathrm{C}(\mathrm{Cl})-\mathrm{C}(\mathrm{Cl})=\mathrm{CH}_{2}\right)$, and 2,3-difluoro-1,3-butadiene $\left(\mathrm{CH}_{2}=\mathrm{C}(\mathrm{F})-\right.$ $\mathrm{C}(\mathrm{F})=\mathrm{CH}_{2}$ ) along the torsional coordinate $\Theta$ calculated using the DSDPBEP86-D3BJ functional. Due to the symmetry of the molecules, the torsional profiles are symmetric with respect to mirroring at $\Theta=180^{\circ}$. The figure shows relative energies $\Delta E$ referenced to the energies of the s-trans structures at $\Theta=180^{\circ}$. (b) Potential energy of 2,3-dibromo-1,3-butadiene as a function of the torsional angle $\Theta$ calculated at CCSD(T)/VTZ-F12 and DSD-PBEP86-D3BJ/def2-QZVPP levels of theory. The lowering of the energy at the local minima due to the zero point vibrational energy ( $\triangle \mathrm{ZPE}$ ) and the Gibbs free energy $(\Delta G)$ is illustrated for the gauche structure. Due to its symmetry, the molecule exhibits two equivalent gauche structures.

The harmonic and anharmonic frequency calculations were performed using DSD-PBEP86-D3BJ as implemented in Gaussian09 using the relevant symmetries. No specific parameters were applied. The DFT results for the torsional potentials and dipole moments were in very good agreement with $\operatorname{CCSD}(\mathrm{T})-\mathrm{F} 12 /$ cc-pVTZ-F12, ${ }^{42-45}$ calculations (Table 1 ). We additionally checked if including Douglas-Kroll-Hess (DKH) scalar relativistic effects on the DFT calculations would effect the results. The dipole moments reduced to $1.9369 \mathrm{D}$ for dibromo-butadiene, $1.9946 \mathrm{D}$ for dichloro-butadiene, and 2.0113 D for difluoro-butadiene, and no change was observed in the cis-trans energy differences upon including DKH corrections. Since DSD-PBEP86-D3BJ results without DKH scalar relaxation effects are closer to the CCSD(T)/VTZ-F12 reference, these corrections were not applied for the results presented here. Furthermore, relaxation at the CCSD/cc-pVTZ-F12 level resulted in geometries identical to those found with DSD-PBEP86-D3BJ/def2-QZVPP with a rootmean-square deviation (RMSD) of $1.9 \mathrm{pm}$ between the final geometries, confirming the reliability of our DFT predictions.

While still exhibiting non-negligible energy differences between the conformers, see Table 1 , the large dipole moment differences among the 2,3-di[halogen]buta-1,3-diene conformers appeared promising, motivating its selection for subsequent experimental investigations. For instance, the dipole-moment difference for 2,3-dibromo-1,3-butadiene was computed at the CCSD(T)/cc-pVTZ-F12 level of theory (neglecting all relativistic effects) to be $\Delta \mu=2.13 \mathrm{D}$.

As the main result of the theoretical screening, 2,3-dibromobuta-1,3-diene (DBB) was identified as an optimal diene for the envisaged experiments that possesses both a sufficiently small energy gap between the gauche and s-trans ground states as well as a large enough difference in the electric dipole moment of the two species (Fig. 2b and Table 2). For gauche-DBB, a dipole moment of $\mu=2.29 \mathrm{D}$ was calculated at the DSD-PBEP86-D3BJ/ def2-QZVPP level of theory, while the s-trans isomer is apolar on grounds of its inversion symmetry. Table 2 summarizes the calculated energy difference as well as the absolute values of the dipole moments and the rotational constants for both conformers.

\subsection{Simulations of the electrostatic deflection of DBB}

Based on the molecular properties obtained from the computations, we predicted trajectories of gauche- and s-trans-DBB molecules through the electrostatic deflector. In Fig. 3, calculated Stark energies (a) and effective dipole moments (b) for rotational states with angular momentum quantum numbers up to $J=2$ of the gauche and s-trans conformers of DBB are shown as a function of electric field strength. In the applied electric fields, all rotational states of the gauche conformer are strong-field seeking with negative Stark shifts, whereas the s-trans conformer does not exhibit a DC Stark effect because of its vanishing dipole moment in the molecular frame. In Fig. 3c, rotational state populations for gauche- and s-trans-DBB at a rotational temperature of $1.0 \mathrm{~K}$ are shown. At this temperature, rotational states up to $J=14$ are significantly populated and can be expected to contribute to the beam-deflection profiles for both conformers.

The density profiles of the molecular beam along the deflection coordinate at the position of intersection with the probe laser (deflection profiles) are plotted in Fig. 3d for the gauche conformer. The color-coded curves show the contributions from the individual rotational states with angular momentum up to $J=20$, while the thick black line corresponds to the total thermally averaged deflection profile at a rotational 
Table 1 Differences in potential energy $\Delta E$ and dipole moment $\Delta \mu$ between the local and global torsional minimas of DBB including thermal corrections at $T=298.15 \mathrm{~K}$ (zero-point and Gibbs free energy) for selected 2,3-dihalogen-substituted butadienes

\begin{tabular}{|c|c|c|c|}
\hline Method & $\mathrm{CH}_{2}=\mathrm{C}(\mathrm{Br})-\mathrm{C}(\mathrm{Br})=\mathrm{CH}_{2}$ & $\mathrm{CH}_{2}=\mathrm{C}(\mathrm{Cl})-\mathrm{C}(\mathrm{Cl})=\mathrm{CH}_{2}$ & $\mathrm{CH}_{2}=\mathrm{C}(\mathrm{F})-\mathrm{C}(\mathrm{F})=\mathrm{CH}_{2}$ \\
\hline & $\Delta E(\mathrm{eV})$ & & \\
\hline CCSD(T)/cc-pVTZ-F12 & 0.097 & 0.117 & 0.151 \\
\hline DSD-PBEP86-D3BJ/def2-QZVPP & 0.097 & 0.114 & 0.155 \\
\hline DSD-PBEP86-D3BJ/def2-QZVPP + harm. therm. corr. & 0.050 & 0.069 & 0.142 \\
\hline \multirow[t]{2}{*}{ DSD-PBEP86-D3BJ/def2-QZVPP + anharm. therm. corr. } & 0.049 & 0.068 & 0.139 \\
\hline & $\Delta \mu$ (Debye) & & \\
\hline CCSD(T)/cc-pVTZ-F12 & 2.1266 & 3.1001 & 2.5380 \\
\hline DSD-PBEP86-D3BJ/def2-QZVPP & 2.2963 & 2.3837 & 2.5938 \\
\hline
\end{tabular}

Table 2 Differences in energy, dipole moments and rotational constants of gauche- and s-trans-2,3-dibromobuta-1,3-diene (DBB) calculated at the DSD-PBEP86-D3BJ/def2-QZVPP level of theory including anharmonic thermal corrections

\begin{tabular}{|c|c|c|c|c|c|}
\hline & \multirow{2}{*}{$\frac{\text { Energy }}{\Delta E=E_{\text {cis }}-E_{\text {trans }}(\mathrm{eV})}$} & \multirow{2}{*}{$\frac{\begin{array}{l}\text { Dipole } \\
\text { moment }\end{array}}{\mu(\mathrm{D})}$} & \multicolumn{3}{|c|}{$\begin{array}{l}\text { Rotational } \\
\text { constants (GHz) }\end{array}$} \\
\hline & & & $A_{\mathrm{e}}$ & $B_{\mathrm{e}}$ & $C_{\mathrm{e}}$ \\
\hline gauche-DBB & 0.049 & 2.29 & 2.3526 & 0.8793 & 0.7097 \\
\hline s-trans-DBB & & 0.00 & 4.6077 & 0.5997 & 0.5306 \\
\hline
\end{tabular}

temperature of $1.0 \mathrm{~K}$. For clarity, the contributions of the individual rotational states have been multiplied by a factor of 4 in the figure. The inset contains the same curves with heights normalised to 1 to allow for a better comparison. The grey area in the main plot is a simulation of the undeflected beam profile, at a deflector voltage of $0 \mathrm{kV}$, which also corresponds to the profile of the apolar s-trans conformer with the deflector turned on. The rotational states of the gauche conformer with largest deflection correspond to small $J$ quantum numbers. Consequently, significant spatial separation of the gauche and s-trans conformers can only be achieved experimentally for samples with a sufficiently low rotational temperature. ${ }^{46}$

\subsection{Experimental deflection profiles}

In order to measure the spatial profiles of the DBB beam emanating from the electrostatic deflector, the molecules were ionized by laser pulses and ejected into the TOF-MS. The choice of the ionization method turned out to be crucial. In Fig. 4a, typical TOF-MS traces obtained using fs-laser-pulse ionization (top trace) and VUV ionization (lower inverted trace) are shown. While fs-laser ionization yielded a large quantity of fragmentation products of the parent DBB molecule, VUV ionization produced a clean mass spectrum with a single peak originating from DBB at $212 \mathrm{u}$. The inset shows an extended mass range around the DBB peak, illustrating that other species or clusters with larger mass cannot be observed under the present experimental conditions.
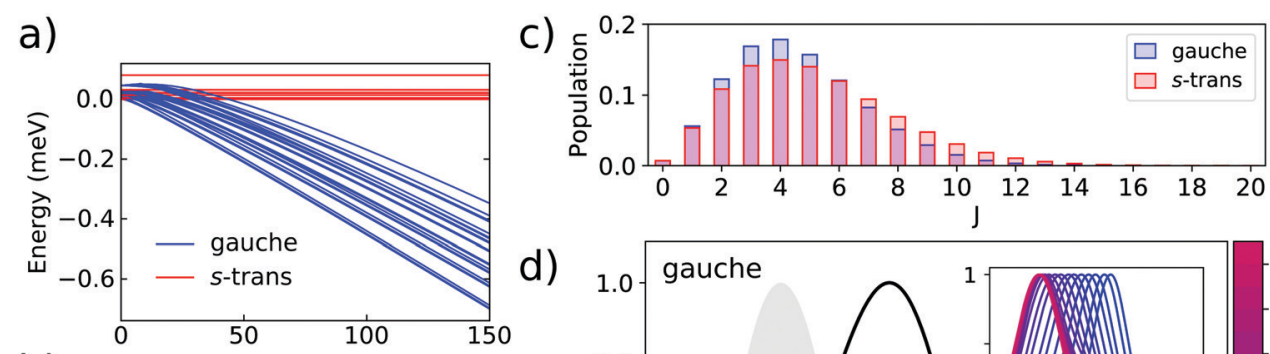

b)
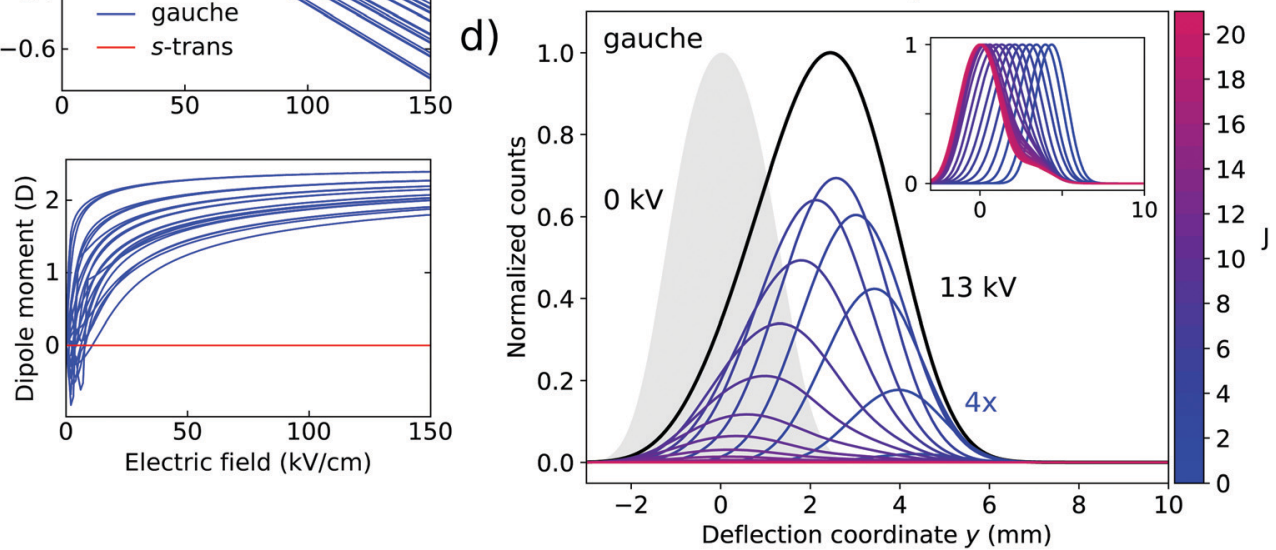

Fig. 3 Simulations. Calculated Stark energies (a) and effective dipole moments (b) vs. electric field strength for individual rotational states with $\mathrm{J}=0,1,2$ of the gauche and s-trans conformers of DBB. (c) Rotational state populations, summed over all levels with the same angular momentum quantum number J, for gauche and s-trans DBB at a rotational temperature of $1.0 \mathrm{~K}$. (d) Simulated deflection profile of the gauche conformer (black line) with its different rotational-state contributions in color. The contributions of the different $J$ states are color-coded according the color scale indicated. The undeflected beam profile is shown by the gray area. 


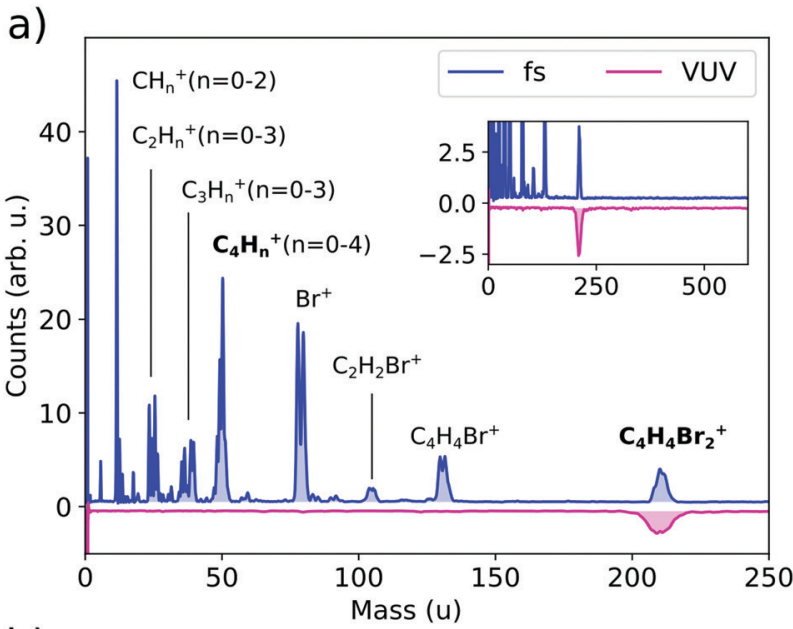

b)

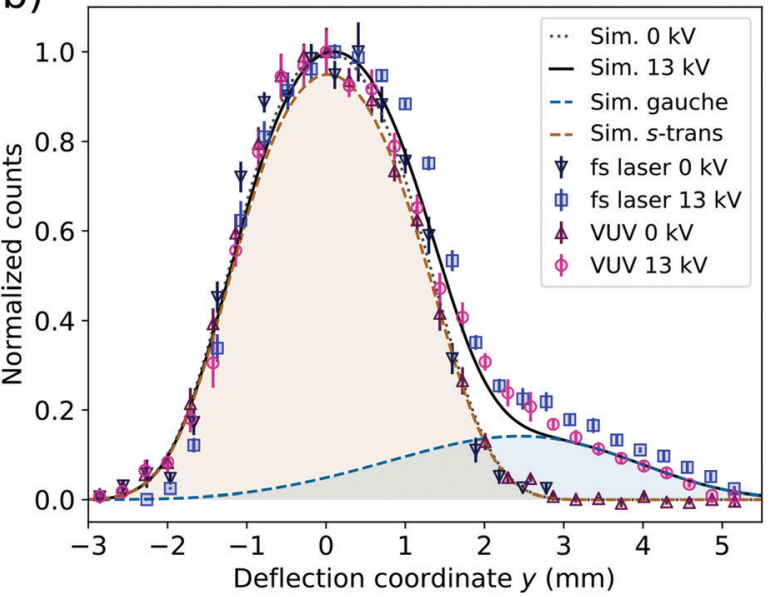

Fig. 4 Deflection profiles. (a) Representative TOF-MS trace obtained using fs-laser-pulse ionization (top trace) and VUV ionization (lower inverted trace) of DBB. (b) Molecular-beam profiles measured using both ionization methods at deflector voltages of $0 \mathrm{kV}$ and $13 \mathrm{kV}$ together with corresponding simulations. For fs-laser-pulse ionization, the profiles for the fragment $\mathrm{C}_{4} \mathrm{H}_{n}{ }^{+}$are shown. Error bars represent standard errors of at least five independent measurements.

Analysis of the different fragment-ion signals obtained from fs-laser ionization, Fig. 5a, revealed that most of the fragments show distinct deflection profiles. Intriguingly, the mass signal corresponding to the parent molecule does not seem to exhibit deflection. This signal could in principle be generated by the break up of larger DBB-containing clusters which may possess only very small dipole moments, similar to the situation observed in the deflection of $\mathrm{H}_{2} \mathrm{O} .{ }^{17}$ However, DBB cluster ions are not observed in the TOF spectra, Fig. 4a, and hence we can essentially rule out that the lack of deflection observed for the $\mathrm{DBB}^{+}$mass peak measured by fs ionization is due to breakup of molecular aggregates. The different deflection profiles recorded for ion signals of the individual fragments are caused by the fs-laserinduced breakup of the parent DBB molecule. It is possible that the electric field of the relatively long laser pulses drives different dynamics for the polar gauche conformer than for the apolar s-trans conformer, thus leading to distinct conformer-specific fragmentation patterns; ${ }^{47}$ these are further discussed on the next page. a)

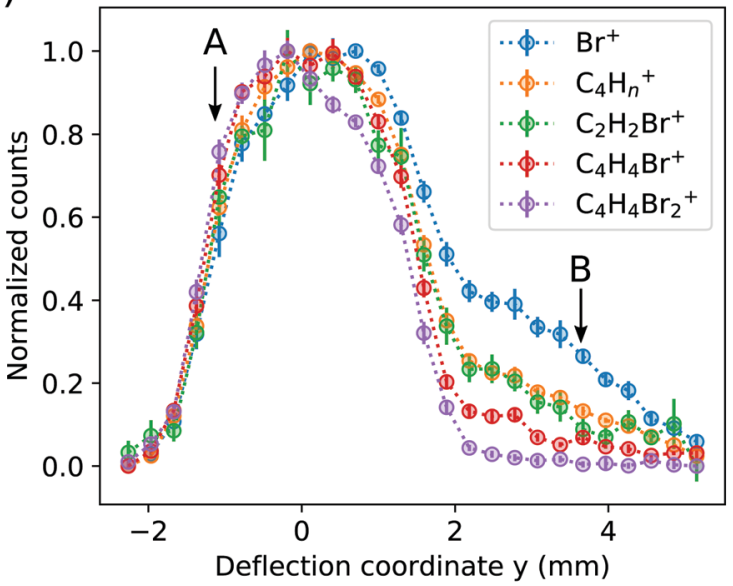

b)

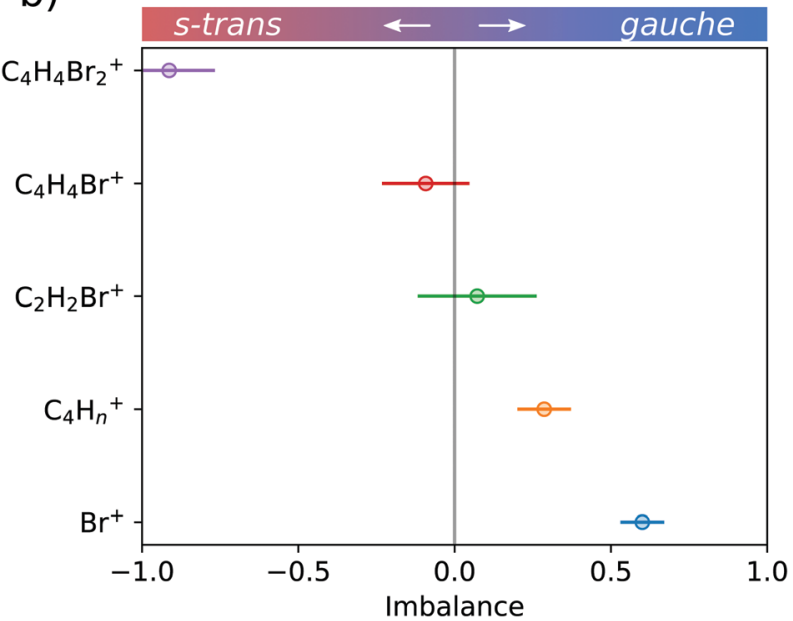

Fig. 5 Deflection curves of a molecular beam containing DBB probed at different ionic fragment masses produced by fs-laser-pulse ionization. (a) lon counts of four molecular fragments and the mass of the parent DBB molecule vs. deflection coordinate. (b) Measured imbalance of fs-laser ionization products between the two points A and B in (a). See text for details. Error bars represent standard errors of six independent measurements.

The complexity of the observed fragmentation dynamics prevented us from unambiguously determining the deflection profile of the DBB parent molecule using fs-laser ionization. Therefore, we implemented soft VUV ionization, which is capable of ionizing DBB without fragmentation as apparent from Fig. 4a and the corresponding molecular-beam profiles in Fig. 4b. While the data points measured with VUV (purple triangles and circles, respectively) probe DBB directly, the data shown for fs-laser-pulse ionization corresponds to the accumulated signal for the fragments $\mathrm{C}_{4} \mathrm{H}_{n}{ }^{+}(n=0 \ldots 4)$ (blue triangles and squares, respectively) produced under these conditions. The experimental data points for VUV ionization agree very well with the simulated thermally averaged beam profiles, which are shown as grey dotted $(0 \mathrm{kV})$ and black solid $(13 \mathrm{kV})$ lines. Corresponding individual contributions from the gauche and s-trans conformers are depicted as the blue and orange shaded areas, respectively. The deflection profile at $13 \mathrm{kV}$ shows a tail 
towards higher deflection coordinates where simulations indicate the presence of pure gauche-DBB. The overall very good agreement between the measured and simulated deflection profiles allows us to confirm the successful separation of the DBB conformers and validates the accuracy of the theoretical calculations.

Further evidence for the separation of the gauche and s-trans conformers can be found in the measured fragmentation products due to fs-laser-pulse ionization of the molecular beam. Fig. 5a shows normalized profiles of four representative fragment families $\mathrm{Br}^{+}, \mathrm{C}_{4} \mathrm{H}_{4}{ }^{+}, \mathrm{C}_{2} \mathrm{H}_{n} \mathrm{Br}^{+}, \mathrm{C}_{4} \mathrm{H}_{4} \mathrm{Br}^{+}$and the parent molecule $\mathrm{DBB}^{+}$as a function of the deflection coordinate. Clearly, the tail of the profile towards large deflection coordinates, where one expects the contribution from gauche-DBB, varies strongly among the different fragments, with $\mathrm{Br}^{+}$showing the largest and $\mathrm{DBB}^{+}$almost zero amplitude. In the region around $y \approx-1 \mathrm{~mm}$, this behavior is inverted. At this location, our trajectory simulations predict a predominance of s-trans-DBB. In order to quantify the imbalance of the observed fragment yields for the gauche and s-trans conformers, we selected the data points at the locations labeled A and B in the figure. From our simulations, we estimate that the populations are $\mathrm{p}_{\text {s-trans }} \approx 1$ at $\mathrm{A}$ and $\mathrm{p}_{\text {strans }} \approx 0$ at $\mathrm{B}$. We evaluate the imbalance between gauche and s-trans for any fragment $\mathrm{X}$ as the relative difference $a_{\mathrm{X}}=\left(n_{\mathrm{X}}^{\mathrm{A}}-n_{\mathrm{X}}^{\mathrm{B}}\right) /\left(n_{\mathrm{X}}^{\mathrm{A}}+n_{\mathrm{X}}^{\mathrm{B}}\right)$ with $n_{\mathrm{X}}^{\mathrm{A}, \mathrm{B}}=N_{\mathrm{X}}^{\mathrm{A}, \mathrm{B}} / N_{\mathrm{VUV}}^{\mathrm{A}, \mathrm{B}}$ being the fragment counts $N_{\mathrm{X}}^{\mathrm{A}, \mathrm{B}}$ normalized by the total DBB beam density $N_{\mathrm{VUV}}^{\mathrm{A}, \mathrm{B}}$ at the respective point as measured by VUV ionization. The imbalance $a_{\mathrm{x}}$ takes values in the range $[-1,+1]$, corresponding to a strong correlation with s-trans or gauche $\mathrm{DBB}$, respectively. Fig. 5b shows the obtained imbalance values which range from -0.9(1) for $\mathrm{DBB}^{+}$ to $0.60(7)$ for $\mathrm{Br}^{+}$. All fragments show a tendency of increasing imbalance towards gauche with decreasing fragment size, thus suggesting that gauche DBB is more likely to break up into smaller parts during the interaction with the fs laser pulse. A rationalization of this phenomenon requires further study.

\section{Conclusions}

Driven by the motivation to gain a precise understanding of the effects of molecular conformation in cycloaddition reactions, a quantum-chemical screening was performed to identify diene candidates suitable for conformer separation in a molecularbeam apparatus. As an optimal diene, 2,3-dibromobuta-1,3diene was found to exhibit the desired large difference in electric dipole moments and small energy difference between the two conformers. Since this particular dihalogenated diene cannot be purchased, mainly due to its intrinsic tendency to undergo polymerization, a synthesis was developed to produce the compound in adequate purity. Experimental validation of the calculated properties was achieved by seeding DBB in a molecular beam and separating its gauche and s-trans conformers in an electrostatic field gradient. A deflection profile of DBB was measured by subsequent ionization and ejection into a time-of-flight mass-spectrometer. The implementation of a vacuum-ultraviolet light source achieved ionization of the parent diene without fragmentation and therefore made it possible to directly measure its deflection behavior. Spatial separation of the two conformers was then confirmed by a close agreement of the observed deflection profiles with Monte-Carlo simulations based on the theoretical molecular properties. Comparison between ion yields from VUV and nonresonant fs-laser-pulse ionization suggests different fragmentation patterns for the gauche and s-trans conformers during ionization in the strong field. The polar gauche conformer showed an enhanced tendency to fragment in comparison with the apolar s-trans conformer. The successful separation of the gauche and s-trans conformers of this tailor-made diene paves the way toward studies of conformer-selected polar cycloaddition reactions in a cold and controlled environment.

\section{Conflicts of interest}

The authors declare no competing financial or non-financial interests.

\section{Acknowledgements}

We thank Philipp Knöpfel, Grischa Martin and Georg Holderried for technical support. Marco Meyer and Jia Wang are acknowledged for their assistance with the experiments. We thank Max Schwilk for fruitful discussions on the theoretical results. This work is supported by the Swiss National Science Foundation (No. BSCGI0_157874). O. A. v. L. acknowledges further funding from the Swiss National Science foundation (No. PP00P2_138932 and 407540_167186 NFP 75 Big Data) and from the European Research Council (ERC-CoG grant QML). This work was partly supported by the NCCR MARVEL, funded by the Swiss National Science Foundation. H. G. and S. W. acknowledge support by the K. C. Wong Education Foundation.

\section{Notes and references}

1 O. Diels and K. Alder, Justus Liebigs Ann. Chem., 1928, 460, 98.

2 K. N. Houk, J. González and Y. Li, Acc. Chem. Res., 1995, 28, 81.

3 J. M. Serafimov, D. Gillingham, S. Kuster and D. Hilvert, J. Am. Chem. Soc., 2008, 130, 7798.

4 L. R. Domingo and J. A. Sáez, Org. Biomol. Chem., 2009, 7, 3576 .

5 L. R. Domingo, J. Chil. Chem. Soc., 2014, 2615, 59.

6 M. A. F. de Souza, E. Ventura, S. A. do Monte, J. M. Riveros and R. L. Longo, J. Comput. Chem., 2016, 37, 701.

7 U. Rivero, O. T. Unke, M. Meuwly and S. Willitsch, J. Chem. Phys., 2019, 151, 104301.

8 D. A. Singleton and A. A. Thomas, J. Am. Chem. Soc., 1995, 117, 9357.

9 P. J. Donoghue and O. Wiest, Chem. - Eur. J., 2006, 12, 7018. 10 U. Rivero, M. Meuwly and S. Willitsch, Chem. Phys. Lett., 2017, 683, 598. 
11 Y.-P. Chang, D. A. Horke, S. Trippel and J. Küpper, Int. Rev. Phys. Chem., 2015, 34, 557.

12 S. Willitsch, Adv. Chem. Phys., 2017, 162, 307.

13 F. Filsinger, U. Erlekam, G. von Helden, J. Küpper and G. Meijer, Phys. Rev. Lett., 2008, 100, 133003.

14 F. Filsinger, J. Küpper, G. Meijer, J. L. Hansen, J. Maurer, J. H. Nielsen, L. Holmegaard and H. Stapelfeldt, Angew. Chem., Int. Ed., 2009, 48, 6900.

15 T. Kierspel, D. A. Horke, Y.-P. Chang and J. Küpper, Chem. Phys. Lett., 2014, 591, 130-132.

16 Y.-P. Chang, K. Dlugolecki, J. Küpper, D. Rösch, D. Wild and S. Willitsch, Science, 2013, 342, 98.

17 A. Kilaj, H. Gao, D. Rösch, U. Rivero, J. Küpper and S. Willitsch, Nat. Commun., 2018, 9, 2096.

18 D. Rösch, S. Willitsch, Y.-P. Chang and J. Küpper, J. Chem. Phys., 2014, 140, 124202.

19 D. A. Horke, Y.-P. Chang, K. Dlugolecki and J. Küpper, Angew. Chem., Int. Ed., 2014, 53, 11965.

20 T. Sperger, I. A. Sanhueza and F. Schoenebeck, Acc. Chem. Res., 2016, 49, 1311.

21 W. Notz, F. Tanaka, S.-i. Watanabe, N. S. Chowdari, J. M. Turner, R. Thayumanavan and C. F. Barbas, J. Org. Chem., 2003, 68, 9624.

22 S. Er, C. Suh, M. P. Marshak and A. Aspuru-Guzik, Chem. Sci., 2015, 6, 885.

23 A. C. Doney, B. J. Rooks, T. Lu and S. E. Wheeler, ACS Catal., 2016, 6, 7948.

24 O. A. von Lilienfeld, Int. J. Quantum Chem., 2013, 113, 1676.

25 M. J. Dewar, S. Olivella and J. J. Stewart, J. Am. Chem. Soc., 1986, 108, 5771.

26 F. Filsinger, S. Putzke, H. Haak, G. Meijer and J. Küpper, Phys. Rev. A: At., Mol., Opt. Phys., 2010, 82, 052513.

27 O. A. von Lilienfeld, in Many-Electron Approaches in Physics, Chemistry and Mathematics, ed. V. Bach and L. D. Site, Springer, 2014, vol. IX, p. 417.

28 S. Kozuch and J. M. L. Martin, Phys. Chem. Chem. Phys., 2011, 13, 20104.

29 F. Weigend and R. Ahlrichs, Phys. Chem. Chem. Phys., 2005, 7, 3297.

30 D. N. Tahchieva, D. Bakowies, R. Ramakrishnan and O. A. von Lilienfeld, J. Chem. Theory Comput., 2018, 14, 4806.

31 M. J. Frisch, G. W. Trucks, H. B. Schlegel, G. E. Scuseria, M. A. Robb, J. R. Cheeseman, G. Scalmani, V. Barone, B. Mennucci, G. A. Petersson, H. Nakatsuji, M. Caricato, X. Li, H. P. Hratchian, A. F. Izmaylov, J. Bloino, G. Zheng, J. L. Sonnenberg, M. Hada, M. Ehara, K. Toyota, R. Fukuda, J. Hasegawa, M. Ishida, T. Nakajima, Y. Honda, O. Kitao,
H. Nakai, T. Vreven, J. A. Montgomery, Jr., J. E. Peralta, F. Ogliaro, M. Bearpark, J. J. Heyd, E. Brothers, K. N. Kudin, V. N. Staroverov, R. Kobayashi, J. Normand, K. Raghavachari, A. Rendell, J. C. Burant, S. S. Iyengar, J. Tomasi, M. Cossi, N. Rega, J. M. Millam, M. Klene, J. E. Knox, J. B. Cross, V. Bakken, C. Adamo, J. Jaramillo, R. Gomperts, R. E. Stratmann, O. Yazyev, A. J. Austin, R. Cammi, C. Pomelli, J. W. Ochterski, R. L. Martin, K. Morokuma, V. G. Zakrzewski, G. A. Voth, P. Salvador, J. J. Dannenberg, S. Dapprich, A. D. Daniels, Ö. Farkas, J. B. Foresman, J. V. Ortiz, J. Cioslowski and D. J. Fox, Gaussian 09 Revision E.01, Gaussian Inc., Wallingford CT, 2009.

32 Y.-P. Chang, F. Filsinger, B. G. Sartakov and J. Küpper, Comput. Phys. Commun., 2014, 185, 339, current version available from https://github.com/CFEL-CMI/cmistark.

33 F. Filsinger, J. Küpper, G. Meijer, L. Holmegaard, J. H. Nielsen, I. Nevo, J. L. Hansen and H. Stapelfeldt, J. Chem. Phys., 2009, 131, 064309.

34 C. A. Stewart Jr., B. Hundred Del and assignor to E. I. du Pont de Nemours and Co, Preparation of 2,3-dichlorobutadiene-1,3, US Pat., 3061653A, 1962.

35 D. Rösch, H. Gao, A. Kilaj and S. Willitsch, EPJ Tech. Instrum., 2016, 3, 5.

36 P. C. Schmid, J. Greenberg, M. I. Miller, K. Loeffler and H. J. Lewandowski, Rev. Sci. Instrum., 2017, 88, 123107.

37 S. M. Hankin, D. M. Villeneuve, P. B. Corkum and D. M. Rayner, Phys. Rev. A: At., Mol., Opt. Phys., 2001, 64, 013405.

38 J. Wiese, J.-F. Olivieri, A. Trabattoni, S. Trippel and J. Küpper, New J. Phys., 2019, 21, 083011.

39 Y. J. Shi, S. Consta, A. K. Das, B. Mallik, D. Lacey and R. H. Lipson, J. Chem. Phys., 2002, 116, 6990.

40 S. E. V. Bramer and M. V. Johnston, Appl. Spectrosc., 1992, 46, 255.

41 R. Steenvoorden, P. Kistemaker, A. D. Vries, L. Michalak and N. Nibbering, Int. J. Mass Spectrom., 1991, 107, 475.

42 H.-J. Werner, G. Knizia and F. R. Manby, Mol. Phys., 2011, 109, 407.

43 K. A. Peterson, T. B. Adler and H.-J. Werner, J. Chem. Phys., 2008, 128, 084102.

44 W. Györffy, G. Knizia and H.-J. Werner, J. Chem. Phys., 2017, 147, 214101.

45 W. Györffy and H.-J. Werner, J. Chem. Phys., 2018, 148, 114104. 46 S. Trippel, M. Johny, T. Kierspel, J. Onvlee, H. Bieker, H. Ye, T. Mullins, L. Gumprecht, K. Długołeęki and J. Küpper, Rev. Sci. Instrum., 2018, 89, 096110.

47 S. Zigo, A.-T. Le, P. Timilsina and C. A. Trallero-Herrero, Sci. Rep., 2017, 7, 42149 EP. 